\title{
Linezolid to treat MDR-/XDR-tuberculosis: available evidence and future scenarios
}

\author{
Giovanni Sotgiu ${ }^{1}$, Emanuele Pontali ${ }^{2}$ and Giovanni Battista Migliori ${ }^{3}$
}

Affiliations: ${ }^{1}$ Clinical Epidemiology and Medical Statistics Unit, Dept of Biomedical Sciences, University of Sassari, Research, Medical Education and Professional Development Unit, Sassari, Italy. ${ }^{2}$ Dept of Infectious Diseases, Galliera Hospital, Genoa, Italy. ${ }^{3}$ World Health Organization Collaborating Centre for Tuberculosis and Lung Diseases, Fondazione S. Maugeri, Care and Research Institute, Tradate, Italy.

Correspondence: Giovanni Battista Migliori, World Health Organization Collaborating Centre for Tuberculosis and Lung Diseases, Fondazione S. Maugeri, Care and Research Institute, via Roncaccio 16, 21049, Tradate, Italy. E-mail: giovannibattista.miglioridafsm.it

@ERSpublications

New experimental evidence on anti-TB regimes based on low-efficacious linezolid dosage to treat XDR-TB patients http://ow.ly/C9YpZ

The figures provided by the World Health Organization (WHO) in its 2013 global tuberculosis (TB) report are remarkable; TB continues to represent a significant clinical and public health problem worldwide [1]. The successes obtained by implementing and scaling-up the WHO public health strategies between 1995 and 2012 (i.e. 22 million lives saved and 56 million patients successfully treated) are outstanding. However, in spite of the dramatic decrease in incidence, prevalence and mortality [1] much needs to be done, and the new WHO post-2015 strategy (which is focused on the concept of TB elimination) is providing further guidance [2-4].

Among the core factors challenging the $\mathrm{TB}$ control and elimination efforts, the most significant are probably the persistent evolution of TB/HIV co-infection and the emergence and spread of drug-resistant TB [1], which has become an unstoppable hurricane [1,5]. The estimated burden of multidrug-resistant (MDR)-TB (i.e. tuberculosis caused by Mycobacterium tuberculosis strains resistant to at least two of the most potent anti-TB drugs, isoniazid and rifampicin) is worrisome. Less than one quarter of the individuals estimated to have MDR-TB were properly diagnosed in 2012; the estimated incidence and mortality are equal to 450000 and 170000 , respectively.

As demonstrated in other infectious diseases, the most significant burden is detected in previously treated rather than newly diagnosed TB cases ( $20 \%$ versus $3.6 \%$, respectively). However, in some settings, mainly located in Eastern Europe and Central Asia, the proportions are further amplified (up to 50\% versus 20\%, respectively) [1, 6-8]. The detection capacity increased between 2011 and 2012 (42\%); although, unfortunately it was not followed by an increased treatment capacity. Out of 94000 MDR-TB patients identified in 2012 only $82 \%$ (equivalent to 77000 patients) were exposed to (appropriate) regimens. Furthermore, almost $10 \%$ of the MDR-TB cohort showed a disease caused by $M$. tuberculosis strains characterised by a worse resistance pattern, known as extensively drug-resistant (XDR)-TB (i.e. MDR-TB with further resistance to any fluoroquinolones and at least one injectable second-line drug, amikacin, capremycin or kanamycin). Its geographical distribution is almost global (at least one case in 92 countries in 2012) [1]. Whenever there was diagnostic capacity it has been inevitably detected.

If we look at the treatment outcomes of these cases, the picture is even worse. Only $48 \%$ of the individuals with MDR-TB belonging to the 2010 cohort were successfully treated worldwide (i.e. microbiologically cured or completed treatment alone), whereas hundreds of patients died or defaulted. Only $31.8 \%$ (34 out of 107) of countries reported an expected treatment success rate $\geq 75 \%$ among their notified MDR-TB cases $[1,9]$.

Received: Aug 072014 | Accepted: Aug 072014

Conflict of interest: None declared.

Copyright OERS 2015 
Three important articles reported the clinical outcomes of the largest MDR-/XDR-TB cohort ever put together and analysed [10-12]. The results of these studies clearly show that the clinical outcomes inevitably become worse when the resistance patterns of the $M$. tuberculosis strains deteriorate $[11,12]$.

Treatment success is lower in patient harbouring XDR-TB strains (40\%) or when resistances to fluoroquinolones are identified (48\%) if compared to patients with MDR-TB isolates (64\%) or harbouring strains that are resistant to second-line injectables alone (56\%). Resistance patterns beyond XDR-TB, involving drugs that are less frequently prescribed, are associated with the poorest outcomes. Treatment success is as low as $30 \%, 34 \%$ and $19 \%$, respectively, in those cases harbouring XDR-TB strains of M. tuberculosis with additional resistance to: 1) amikacin/kanamycin and capreomycin; 2) XDR-TB plus resistance to kanamycin and at least one WHO group 4 medicine; and 3) XDR-TB plus resistance to kanamycin, at least one WHO group 4 medicine, pyrazinamide and/or ethambutol [10-12].

The suggested remedy to these catastrophic clinical conditions is represented by regimens based on a significant load of second- and third-line drugs in the intensive (six medicines), as well as the continuation (four medicines), phase of treatment. Furthermore, the overall treatment duration, particularly in the more severe forms, should not be inferior to 20 months, with the intensive phase being $>6.5$ months $[11,13]$.

In spite of the progress achieved in diagnosing TB and MDR-TB (e.g. GeneXpert (Cepheid, Sunnyvale, CA, USA), although other diagnostics are in the pipeline for the global scale-up) several (unlucky) patients are still treated in a pre-antibiotic scenario [14, 15].

For more than 40 years, no new TB-specific antibiotic has been launched in the treatment arena until recently when delamanid and bedaquiline finally appeared on the horizon [16-22]. Although their appearance is generating obvious enthusiasm, they are not able to solve all the MDR-/XDR-TB management problems per se.

The necessary new, evidence-based, clinical and public health approach to MDR-/XDR-TB implies a stewardship of new and old medicines, as well as a fight against markets and practices based on non-quality-assured drugs and insufficient supplies. Furthermore, we need to tackle all the clinical, social and epidemiological factors associated with poor patient adherence. Availability of shorter regimens based on more potent bactericidal drugs is a pre-requisite to achieve real progress [23-26].

The few available alternatives to the first-line anti-TB drugs are less efficacious, more toxic and more expensive [1]. However, the true efficacy, safety and tolerability profile of some of these antibiotics, particularly those belonging to the WHO category 5 (e.g. linezolid, meropenem, cotrimoxazole, etc.), was rarely explored in adequately designed, randomised, controlled, multicentre clinical trials [27, 28].

In the majority of cases, the available evidence is composed of inadequately or not designed observational, mono-centre studies or case reports, where the enrolment is either consecutive or based on the clinical needs of the physician in charge. In the most fortunate cases, systematic reviews are available. Nevertheless, this does not represent the gold standard evidence the modern evidence-based medicine aims for, and the risk of biases (with resulting unreliable findings) are highest.

The story of linezolid is, in a way, instructive. After the appearance of the first collection of in vitro data, the drug was used "off-label" even before a sufficient amount of preliminary evidence from case series and multicentre observational studies was made available [29]. Only at a later stage were systematic (individual and nonindividual) reviews and new experimental support to the definition of its profile finally published [29-34].

To better describe the story of this drug, we looked at the evidence available on linezolid efficacy, safety and tolerability following a PubMed search. A nonsystematic review was carried out using the keywords "linezolid" and "tuberculosis" to detect the observational and the experimental studies conducted by July 2014 in the adult population (i.e. no time restrictions). The identified studies are summarised in tables 1 and 2 and briefly described below.

The most striking feature of the clinical trials conducted on linezolid to date is the confirmation of previous observational findings [31, 32]. A systematic review and meta-analysis based on individual data, collected from 12 studies performed in 11 countries globally, showed a relevant efficacy of the linezolid-containing regimens in treating MDR-TB patients. Sputum-smear- and -culture conversions were achieved in $92.5 \%$ and $93.5 \%$, respectively, with a treatment success rate of $\sim 82 \%$ [31]. Unfortunately, its safety and tolerability profile was not favourable; $58.9 \%$ experienced adverse events (particularly haematological, neurological and gastrointestinal), mainly in the cohort of individuals treated with an oral daily dosage $>600 \mathrm{mg}$. The experimental study of LeE et al. [32] was carried out in 39 XDR-TB patients, $89 \%$ of whom achieved sputum-culture conversion after 6 months of exposure due to a daily linezolid dose of $600 \mathrm{mg}$ within individualised background regimens, based on WHO guidelines. 
TABLE 1 Efficacy of linezolid containing regimens in multidrug-resistant tuberculosis (MDR-TB) adults: the current scientific evidence

\begin{tabular}{|c|c|c|c|c|c|c|}
\hline First author [ref.] & $\begin{array}{l}\text { Sample } \\
\text { size }\end{array}$ & Design & $\begin{array}{l}\text { MDR-/ } \\
\text { XDR-TB }\end{array}$ & $\begin{array}{l}\text { Culture } \\
\text { conversion }\end{array}$ & $\begin{array}{l}\text { Treatment } \\
\text { success }\end{array}$ & $\begin{array}{l}\text { Daily linezolid } \\
\text { dosage mg }\end{array}$ \\
\hline \multicolumn{7}{|c|}{ Observational studies } \\
\hline ZHANG [35] & 15 & Retrospective & $0 / 15$ & $9 / 15(60.0)$ & $9 / 15$ (60.0) & 600 once daily \\
\hline$X u[36]$ & 18 & Retrospective & $3 / 15$ & $16 / 18[88.9]$ & $9 / 18(50.0)$ & 600 twice daily, 900 \\
\hline TANG [34] & 65 & Randomised, controlled & $0 / 65$ & 26/33 (78.8) & 23/33 (69.7) & $\begin{array}{l}\text { 300, } 600 \text { lonce and } \\
\text { twice daily) }\end{array}$ \\
\hline
\end{tabular}

Data are presented as $\mathrm{n}$ or $\mathrm{n} / \mathrm{N}(\%)$. XDR-TB: extensively drug-resistant tuberculosis.

\begin{tabular}{|c|c|c|c|c|c|c|}
\hline First author [ref.] & Adverse events & Discontinuation rate & Anaemia & Neuropathy & GI events & Duration of exposure days \\
\hline \multicolumn{7}{|c|}{ Observational studies } \\
\hline SotGIU [31] & 63/107 (58.9) & $54 / 79(68.4)$ & $32 / 84(38.1)$ & 40/85 (47.1) & 14/84 (16.7) & $300^{\#}$ \\
\hline ZHANG [35] & $6 / 15(40.0)$ & & $4 / 15(26.6)$ & $1 / 15(6.7)$ & $3 / 15(20.0)$ & $60^{\#}$ \\
\hline $\mathrm{Xu}[36]$ & $17 / 18(94.4)$ & & $12 / 18(66.7)$ & $11 / 18(61.1)$ & $15 / 18(83.3)$ & $180^{\circ}$ \\
\hline LEE [32] & 33/38 (87) & $3 / 38(7.9)^{+}$ & $7 / 38(18.4)$ & $21 / 38(55.3)$ & & At least 180 \\
\hline TANG [34] & 27/33 (81.8) & $2 / 33(6.1)^{+}$ & $17 / 33(51.5)$ & $8 / 33(24.2)$ & $16 / 33(48.5)$ & $360^{9}$ \\
\hline
\end{tabular}

Data are presented as n/N (\%), unless otherwise stated. GI: gastrointestinal. " ${ }^{\text {: }}$ median; ${ }^{\text {ๆ: }}$ mean; ${ }^{+}$: permanent.

Furthermore, a group of patients was successfully treated with a lower dose $(300 \mathrm{mg})$ of linezolid. The proportion of adverse events was $>80 \%$ but was less elevated in the group of individuals exposed to $300 \mathrm{mg}$ once a day $[32,33]$.

In the current issue of the European Respiratory Journal, an important experimental study is described. TANG et al. [34] carried out a randomised, controlled clinical trial, recruiting 65 XDR-TB patients who started individualised anti-TB regimens containing linezolid (600 mg twice a day) for $\sim 1$ month and were then shifted to $600 \mathrm{mg}$ or $300 \mathrm{mg}$ once a day. Linezolid was prescribed to 33 patients. After 2 years the proportion of sputum-culture conversion was outstanding in those exposed to linezolid-containing regimens when compared with those in the control arm (78.8\% versus $37.6 \%)$. Furthermore, the treatment success rates achieved (69.7\% and 51.5\%, respectively, were microbiologically cured) were $>30 \%$ higher in comparison with the estimates of the systematic review based on observational studies [31]. Unfortunately, the safety and tolerability profile continues to represent the negative face of linezolid administration, with $82 \%$ of the patients experiencing adverse events (which fortunately are transient in the majority of cases).

Hence, recent evidence, particularly on patients affected by XDR-TB, provides new perspectives and new hopes to the patients and their families for survival. While delamanid and bedaquiline are gradually taking a definite role in the treatment armamentarium [17-22, 37], physicians finally have access to more and more evidence for the correct use of linezolid in order to use it in the manufacture of appropriate and successful regimens for those patients in need.

However, the linezolid profile is not yet completely known. Quality clinical research is needed to better understand the appropriate dosage that physicians can prescribe to minimise the probability of adverse reactions. Recent pharmacological studies, based on the therapeutic drug monitoring approach, seem to achieve a better efficacy/tolerability compromise (fig. 1) [30]. Notably, in the meta-analysis mentioned above [31], the clinical centre in the Netherlands performing systematically therapeutic drug monitoring 


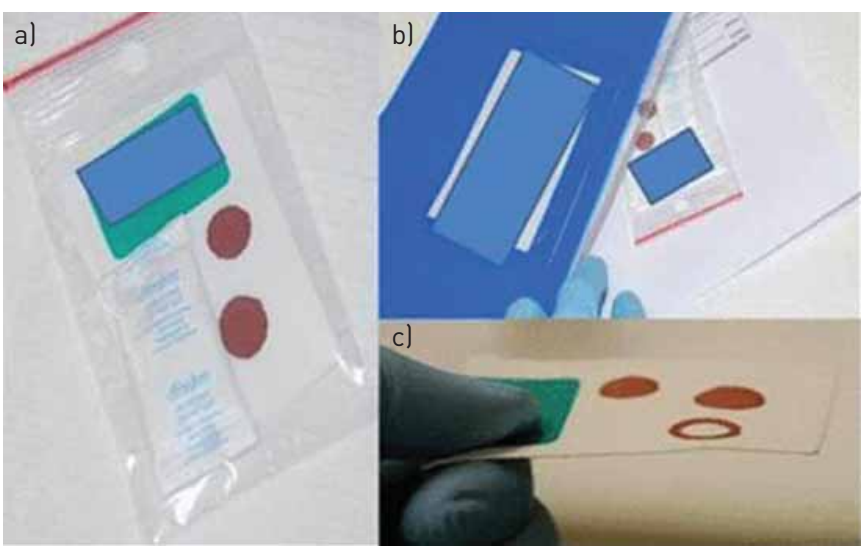

FIGURE 1 Dried blood spot (DBS) for therapeutic drug monitoring. Blood is collected on paper strips (a) and packed in a plastic bag with a desiccant to keep the strip dry. Samples can then be transported (b) via regular post or any other suitable means. The DBS is collected from the strip and the drug is extracted and concentrations subsequently measured using validated methods. Reproduced from [38] with permission from the publisher.

on linezolid reports a proportion of adverse events far below the average ( $0 \%$ undergoing therapeutic drug monitoring versus $59 \%$ not undergoing monitoring).

Nevertheless, the greatest limit to this approach is the paucity of laboratories that are able to perform linezolid therapeutic drug monitoring on a routine basis in countries most affected by MDR-/XDR-TB.

We are convinced that regimens tailored to the individuals' metabolism will play a noteworthy role to reduce linezolid-related toxicity and further enhance the role of this drug in managing cases with resistance beyond XDR-TB.

According to the available information, bedaquiline and delamanid seem to be effective and well tolerated, pending confirmation from existing trials [17-22]. They might favour the design of new, shorter and effective regimens that are able to treat both drug-susceptible TB and MDR-TB cases.

What will be the role of linezolid in this new scenario? As a significant burden of MDR-/XDR-TB already exists, particularly in Former Soviet Union countries (up to $50 \%$ of the cases and one out of three newly detected cases $[1,8]$ ), we need to be prepared to use a sufficient number of drugs within WHO category 5 to treat cases with resistance beyond XDR-TB.

The combination of linezolid and the other anti-TB drugs in the evidence-based regimens are expensive [39], but at present neither bedaquiline nor delamanid can be considered to be cheap [40, 41].

Further research is needed to better understand appropriate conditions where linezolid-containing regimens will be able to perform best (patient sub-groups, geographical settings, $M$. tuberculosis resistance patterns, concomitant drugs in the regimen, etc.).

A final comment is that on top of studying new drugs we need public health research and support for health systems and TB programmes. After 40 years of neglect we finally have a few new drugs, and we are gradually understanding the role that other re-proposed drugs (linezolid, meropenem and cotrimoxazole among others $[27,28,31])$ might have in managing difficult-to-treat TB cases. We cannot afford to lose them in a much shorter time than that necessary for their development. In our hands, the risk is that this new pharmacological sunrise falls into a déjà $v u$ pharmacological sunset.

\section{References}

World Health Organization. Global Tuberculosis Report 2013. Geneva, WHO, 2013.

2 Sotgiu G, Mauch V, Migliori GB, et al. Evidence-based, agreed-upon health priorities to remedy the tuberculosis patient's economic disaster. Eur Respir J 2014; 43: 1563-1566.

3 Voniatis C, Migliori GB, Voniatis M, et al. Tuberculosis elimination: dream or reality? The case of Cyprus. Eur Respir J 2014; 44: 543-546.

4 Sotgiu G, Migliori GB. Is tuberculosis elimination a reality? Lancet Infect Dis 2014; 14: 364-365.

5 Falzon D, Zellweger J-P, Migliori GB, et al. Drug-resistance and tuberculosis elimination in low-incidence countries. Eur Respir J 2014; 44: 1408-1411.

6 Zignol M, Dara M, Dean AS, et al. Drug-resistant tuberculosis in the WHO European Region: an analysis of surveillance data. Drug Resist Updat 2013; 16: 108-115.

7 Ulmasova DJ, Uzakova G, Tillyashayhov MN, et al. Multidrug-resistant tuberculosis in Uzbekistan: results of a nationwide survey, 2010 to 2011. Euro Surveill 2013; 18.

8 Skrahina A, Hurevich H, Zalutskaya A, et al. Alarming levels of drug-resistant tuberculosis in Belarus: results of a survey in Minsk. Eur Respir J 2012; 39: 1425-1431.

9 Falzon D, Jaramillo E, Wares F, et al. Universal access to care for multidrug-resistant tuberculosis: an analysis of surveillance data. Lancet Infect Dis 2013; 13: 690-697. 
10 Ahuja SD, Ashkin D, Avendano M, et al. Multidrug resistant pulmonary tuberculosis treatment regimens and patient outcomes: an individual patient data meta-analysis of 9,153 patients. PLoS Med 2012; 9: e1001300.

11 Falzon D, Gandhi N, Migliori GB, et al. Resistance to fluoroquinolones and second-line injectable drugs: impact on multidrug-resistant TB outcomes. Eur Respir J 2013; 42: 156-168.

12 Migliori GB, Sotgiu G, Gandhi NR, et al. Drug resistance beyond extensively drug-resistant tuberculosis: individual patient data meta-analysis. Eur Respir J 2013; 42: 169-179.

13 Falzon D, Jaramillo E, Schünemann HJ, et al. WHO guidelines for the programmatic management of drug-resistant tuberculosis: 2011 update. Eur Respir J 2011; 38: 516-528.

14 Migliori GB, Sotgiu G, Lange C, et al. Extensively drug-resistant tuberculosis: back to the future. Eur Respir J 2010; 36: 475-477.

15 Hanson C, Sotgiu G, Loddenkemper R. Ensuring that the diagnosis of tuberculosis accelerates progress towards the Millennium Development Goals. Eur Respir J 2014; 44: 1-4.

16 Raviglione M, Marais B, Floyd K, et al. Scaling up interventions to achieve global tuberculosis control: progress and new developments. Lancet 2012; 379: 1902-1913.

17 Zumla AI, Gillespie SH, Hoelscher M, et al. New antituberculosis drugs, regimens, and adjunct therapies: needs, advances, and future prospects. Lancet Infect Dis 2014; 14: 327-340.

18 Kwon YS, Jeong BH, Koh WJ. Tuberculosis: clinical trials and new drug regimens. Curr Opin Pulm Med 2014; 20: 280-286.

19 Skripconoka V, Danilovits M, Pehme L, et al. Delamanid improves outcomes and reduces mortality in multidrug-resistant tuberculosis. Eur Respir J 2013; 41: 1393-1400.

20 Tiberi S, De Lorenzo S, Centis R, et al. Bedaquiline in MDR/XDR-TB cases: first experience on compassionate use. Eur Respir J 2014; 43: 289-292.

21 van Halsema C, Humphreys S, Bonington A. Extensively drug-resistant tuberculosis: early access to bedaquiline for a UK patient. Eur Respir J 2014; 43: 292-294.

22 Diacon AH, Donald PR, Pym A, et al. Randomized pilot trial of eight weeks of bedaquiline (TMC207) treatment for multidrug-resistant tuberculosis: long-term outcome, tolerability, and effect on emergence of drug resistance. Antimicrob Agents Chemother 2012; 56: 3271-3276.

23 Elzinga G, Raviglione MC, Maher D. Scale up: meeting targets in global tuberculosis control. Lancet 2004; 363: 814-819.

24 Raviglione MC, Lange C, Migliori GB. Preventing and managing antimicrobial resistance: imperative for chest physicians. Eur Respir J 2011; 37: 978-981.

25 Gospodarevskaya E, Tulloch O, Bunga C, et al. Patient costs during tuberculosis treatment in Bangladesh and Tanzania: the potential of shorter regimens. Int J Tuberc Lung Dis 2014; 18: 810-817.

26 Fofana MO, Knight GM, Gomez GB, et al. Population-level impact of shorter-course regimens for tuberculosis: a model-based analysis. PLoS One 2014; 9: e96389.

27 De Lorenzo S, Alffenaar JW, Sotgiu G, et al. Efficacy and safety of meropenem-clavulanate added to linezolid-containing regimens in the treatment of MDR-/XDR-TB. Eur Respir J 2013; 41: 1386-1392.

28 Alsaad N, van Altena R, Pranger AD, et al. Evaluation of co-trimoxazole in the treatment of multidrug-resistant tuberculosis. Eur Respir J 2013; 42: 504-512.

29 Migliori GB, Eker B, Richardson MD, et al. A retrospective TBNET assessment of linezolid safety, tolerability and efficacy in multidrug-resistant tuberculosis. Eur Respir J 2009; 34: 387-393.

30 Cox H, Ford N. Linezolid for the treatment of complicated drug-resistant tuberculosis: a systematic review and meta-analysis. Int J Tuberc Lung Dis 2012; 16: 447-454.

31 Sotgiu G, Centis R, D'Ambrosio L, et al. Efficacy, safety and tolerability of linezolid containing regimens in treating MDR-TB and XDR-TB: systematic review and meta-analysis. Eur Respir J 2012; 40: 1430-1442.

32 Lee M, Lee J, Carroll MW, et al. Linezolid for treatment of chronic extensively drug-resistant tuberculosis. $N$ Engl J Med 2012; 367: 1508-1518.

33 Sotgiu G, Centis R, D'Ambrosio L, et al. Linezolid to treat extensively drug-resistant TB: retrospective data are confirmed by experimental evidence. Eur Respir J 2013; 42: 288-290.

34 Tang S, Yao L, Hao X, et al. Efficacy, safety and tolerability of linezolid for the treatment of XDR-TB: a study in China. Eur Respir J 2015; 45: 161-170.

35 Zhang L, Pang Y, Yu X, et al. Linezolid in the treatment of extensively drug-resistant tuberculosis. Infection 2014; 42: 705-711.

$36 \mathrm{Xu} \mathrm{HB}$, Jiang $\mathrm{RH}, \mathrm{Li} \mathrm{L}$, et al. Linezolid in the treatment of MDR-TB: a retrospective clinical study. Int $J$ Tuberc Lung Dis 2012; 16: 358-363.

37 Esposito S, D’Ambrosio L, Tadolini M, et al. ERS/WHO Tuberculosis Consilium assistance with extensively drug-resistant tuberculosis management in a child: case study of compassionate delamanid use. Eur Respir J 2014; 44: $811-815$

38 Srivastava S, Peloquin CA, Sotgiu G, et al. Therapeutic drug management: is it the future of multidrug-resistant tuberculosis treatment? Eur Respir J 2013; 42: 1449-1453.

39 Diel R, Vandeputte J, de Vries G, et al. Costs of tuberculosis disease in the European Union: a systematic analysis and cost calculation. Eur Respir J 2014; 43: 554-565.

40 Migliori GB, Lienhardt C, Weyer K, et al. Ensuring rational introduction and responsible use of new TB tools: outcome of an ERS multisector consultation. Eur Respir J 2014; 44: 1412-1417.

41 World Health Organization. The use of bedaquiline in the treatment of multidrug-resistant tuberculosis. Interim policy guidance. Geneva, WHO, 2013. 